\title{
RADIATION INDUCED SOFT FAILS IN SPACE ELECTRONICS*
}

\author{
E. L. Petersen \\ Naval Research Laboratory \\ Washington, D. C. 20375
}

\section{Abstract}

Advanced microelectronic circuits are sensitive to the passage of single ionizing particles. These circuits store information so that the deposition of a sma11 charge (picocoulomb range) can charge a storage node, resulting in a bit error. This effect presents a problem to computer memories on earth because alpha particles are emitted from microelectronic packages and materials. The problem is much more severe in space due to primary cosmic rays and to nuclear reactions in the device produced by protons from the earth's radiation belts. A number of satellite systems have been severely affected by single event soft-fails.

\section{Int'roduction}

In the 1970's anomalies were observed in the operation of several spacecraft. A paper was presented in 1975 proposing that memory upsets were caused by the passage of cosmic rays. ${ }^{1}$ This idea was ignored until May and Woods ${ }^{2}$ showed that temporary (soft) single bit failures in commercial $16 \mathrm{~K}$ dynamic RAMs were caused by alpha particles from radioactive impurities. Interest in single event radiation effects has increased as more and more circuits have been found to be susceptible. The effects are proving to be a severe problem in the space environment due both to cosmic rays and to the trapped protons in the Van Allen belt. Many satellite systems have had problems that can be explained by the effects of single nuclear particles.

\section{SINGLE EVENT UPSET MECHANISM CMOS STATIC RAM}
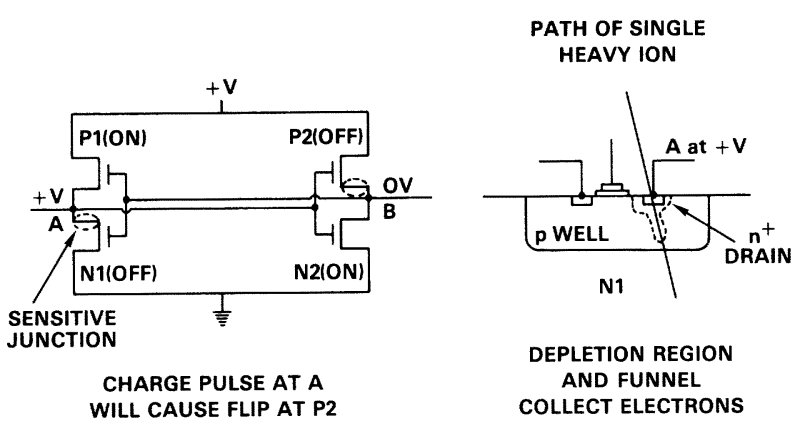

\section{DEPLETION REGION \\ AND FUNNEL}

COLLECT ELECTRONS

Figure 1. A simple flip-flop circuit. The passage of a charged particle can cause it to flip, leading to a temporary error in the stored data.

*This research was partially funded by NAVELEX under the NRL Nuclear Survivability/Vulnerability Program and by the DNA/DARPA Single Event Radiation Effects Program.
Single event upsets have been made possible by the electronics industry in its push to increase the speed and reduce the power of integrated circuits. Figure 1 shows a typical flip-flop circuit used to store binary information. A cosmic ray passing through the device leaves a trail of positive and negative charges. If the path is through a depletion region, part of these charges will be collected. As indicated schematically in Figure 2, the depletion region is extended into the substrate by the recently discovered funnel effect. 3 The resulting voltage on that node appears exactly as if a signal had been injected. Single event upsets are possible when the charge collected is greater than the critical charge, $Q_{c}$, necessary to cause a change of state. The critical charges for modern devices are in the range $0.05 \mathrm{pC}$ to 1 pC. Figure 2 shows the charge deposition in $10 \mu \mathrm{m}$ of silicon as a function of energy for various ions. 4 clearly, there is a potential for both cosmic rays and nuclear reaction products to cause upsets.

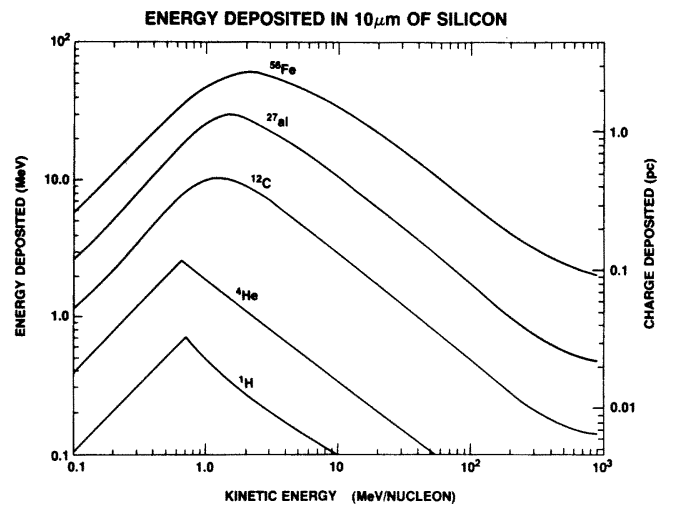

Figure 2. Energy and charge deposited in $10 \mu \mathrm{m}$ of silicon by various ions. The straight part of the curves at low energy occurs when the ion range is less than $10 \mu \mathrm{m}$ and it deposits a11 of its energy.

\section{The Space Environment}

Present spacecraft operate in two distinct regions of space: a lower region between 100 and $600 \mathrm{nmi}$. (185-1111 km) and an upper region above 6,000 nmi. Future spacecraft may operate in the intermediate region between 600 and $6,000 \mathrm{nmi}$, in the intense proton belts. The radiation environment in the outer region consists of electrons (to well outside geostationary orbit) and cosmic rays. The cosmic ray population consists of galactic cosmic rays and various particles associated with the solar system. 5 For single event upset consideration, it is useful to consider the particle population as a function of its energy loss (Figure 3) rather than in terms of energy and particle species. 6 Figure 3 shows the energy loss spectrum for best, moderate, and worst case situations, as well as spectra that have been used for two sub-geosynchronous orbits. 7 


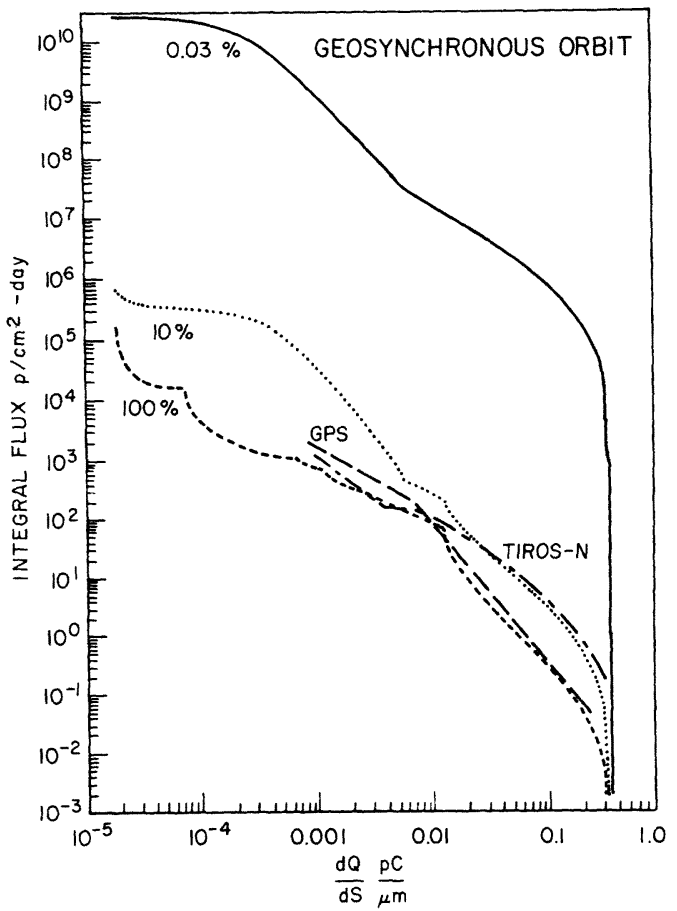

Figure 3. Integral energy loss spectrum at geosynchronous orbit. The 100 percent curve is the best case situation, while the environment is only worse than the 10 percent curve 10 percent of the time. The 0.03 percent curve corresponds to a large solar flare. The curves labeled GPS and TIROS-N, were used to calculate upset rates for those two sub-geosynchronous missions.

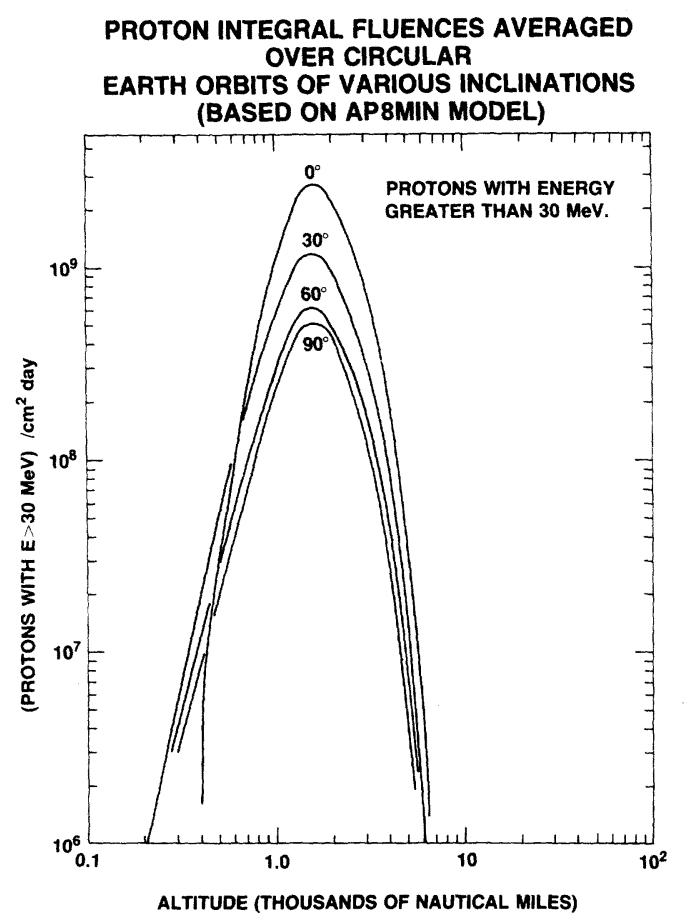

Figure 4. Proton fluences as a function of altitude and inclination in the radiation belt.
Protons are not likely to cause upsets by direct ionization. However, the proton radiation belt is sufficiently populated so that proton induced reactions in the device are a problem. Figure 4 shows the time averaged fluences of high energy protons that an orbiting spacecraft would encounter. These protons can penetrate deeply into a satellite. Figure 5 shows the trapped proton spectrum for a $600 \mathrm{nmi}(1111 \mathrm{~km})$ orbit. The shape of the spectrum changes as the protons lose energy passing into the spacecraft. The dashed curves show the flux after passing through various thicknesses of concentric shell. The inner solid curve shows the resulting spectrum after the incident flux passes through the shielding presented by a typical light spacecraft.

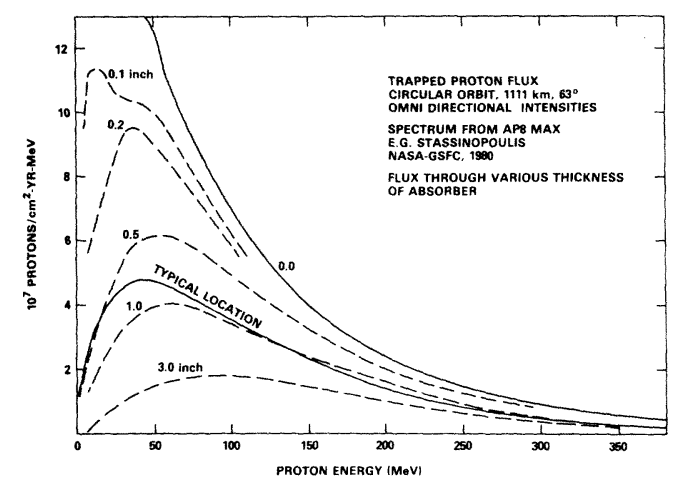

Figure 5. The omnidirectional trapped flux for a $600 \mathrm{nmi}$ orbit. The dashed curves show the flux inside various concentric shells. The inner solid curve shows the resulting spectrum after the incident flux passes into a typical light spacecraft.

\section{Upset Rates in Orbit}

The proton induced upsets are caused primarily by the heavy recoiling particles produced in nuclear reactions in the silicon device. Spallation reactions can deposit the most energy, but have relatively low cross sections. The reactions producing alpha particles or protons deposit less energy. It is possible to estimate upset rates in a device by considering the nuclear reaction cross sections and energetics. Figure 6 shows the upset rate estimated as a function of device sensitivity for the spectrum obtained in Figure 5 and an arbitrarily chosen device volume.

The cosmic ray upset rate can be calculated by combining the energy loss spectrum of Figure 3 with the path length distribution of a given device. In general this gives good agreement with the upset rates observed in space. 7,9

\section{Future Upset Rates}

Simple considerations indicate that the critical charge for upset decreases rapidly as device size decreases.6,10 This implies that much larger fractions of the cosmic ray population can induce upset. This then suggests the possibility that upset rates might increase drastically as devices become smaller. Figure 7 shows the dependance of critical charge on device feature size ( $l$ ) for a number of devices ${ }^{6}$, supporting the assumption that $Q_{c}$ is proportional to $\ell^{2}$. This scaling rule 


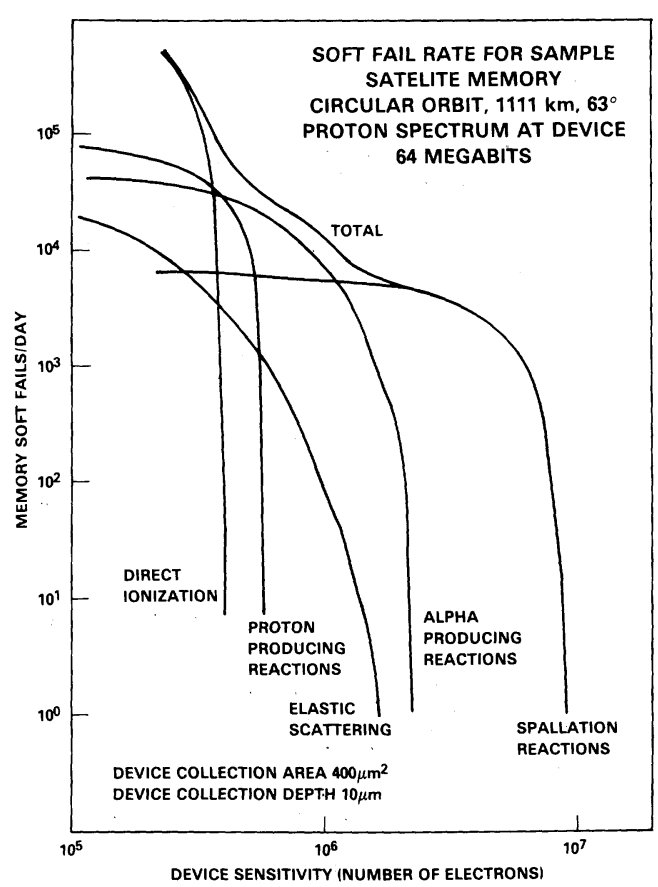

Figure 6. Upset rate in a sample satellite memory as a function of device sensitivity. These rates are for the trapped proton spectrum obtained in Figure 5.

applies either in bulk devices where all dimensions are scaled down uniformly, or in surface devices where the depth is not scaled. Figure 8 shows the predicted upset rate ${ }^{6}$ for two devices assuming that $Q_{c}$ scales as in Figure 7. A calculation starting with a more sensitive bulk device and a less sensitive surface device has the same character, but the two curves do not cross 10 . In any case, the upset rates do not become catastrophic as devices are scaled down.

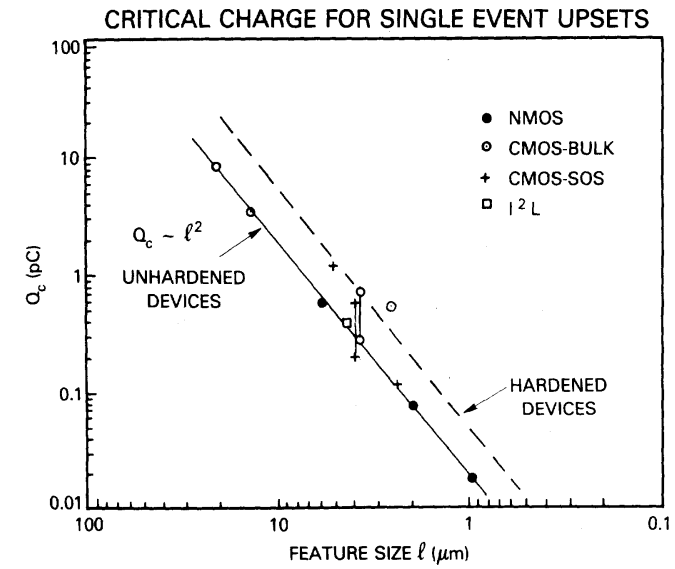

Figure 7. Critical charge for upset plotted as a function of feature size. There is surprisingly little dependence on device technology. It is possible to move away from these curves by modifying the standard circuits.

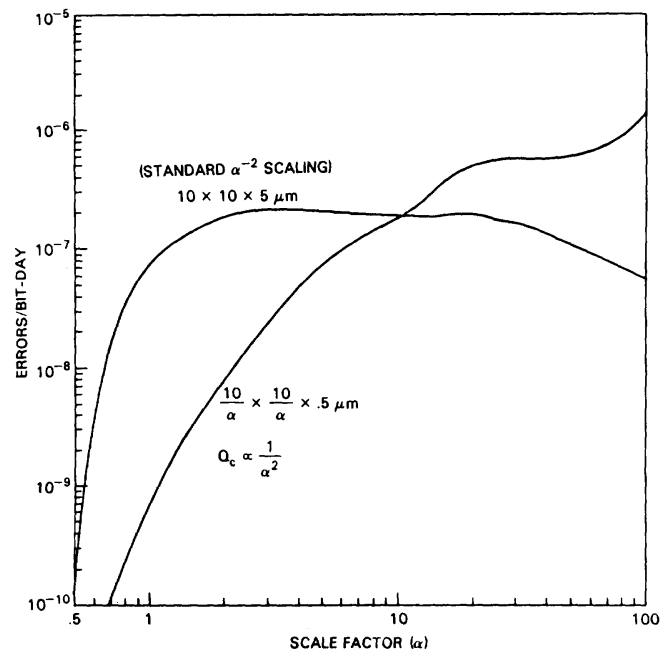

Figure 8. Upset rates plotted as a function of scale factor. The $10 \times 10 \times 5 \mu \mathrm{m}$ device (at a scale factor $=1$ ) scales all dimensions by the factor a while the $10 \times 10 \times 0.5 \mu \mathrm{m}$ device is assumed to keep a constant thickness. The critical charge for both devices follows the unhardened curve of Figure 7 . These rates are for the 10 percent worst case cosmic ray spectrum shown in Figure 3.

Figures 6 and 8 both show that upset rates have a rapid increase if a device threshold is passed. There are currently a number of devices that upset for cosmic rays, but not for proton induced reactions. Figure 9 examines threshold behavior for proton induced upsets as devices scale. The curves compare the maximum charge that could be deposited in a path length $(l)$ and the threshold critical charge as a function of feature size $(l)$. It appears that most devices will upset to protons as their feature size is scaled down to one micron.

SINGLE EVENT UPSET THRESHOLD

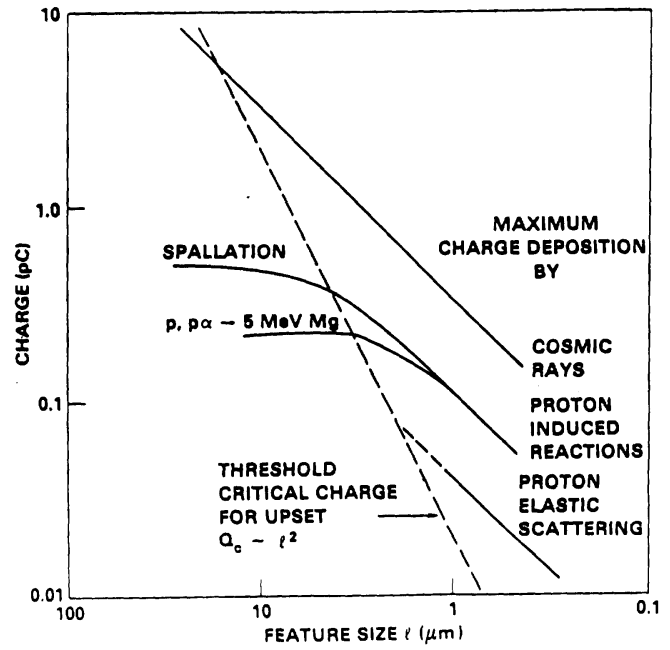

Figure 9. Single Event Threshold $\left(Q_{c}\right)$ plotted as a function of feature size (--). The solid curves show the maximum charge deposition in a distance $l$ for several processes. If these values exceed the threshold charge, single event upsets may happen. 
The space environment is one of the few places in nature where nuclear reactions and other properties of individual nuclei can have a drastic effect on man and his work. Single event soft failures of microelectronics have become a serious problem for present microelectronic devices in space, and promise to continue to be a problem as electrical devices continue to shrink.

\section{References}

1. D. Binder, E.C. Smith, and A.B. Holman, "Satellite Anomalies from Galactic Cosmic Rays," IEEE Trans. Nuc1. Sci., NS-22, 2675 (1975).

2. T.C. May and M.H. Wood, "Alpha-Particle-Induced Soft Errors in Dynamic Memories," IEEE Trans. Elec. Dev., ED-26, 2 (1979).

3. C.M. Hsieh, P.C. Murley, and R.R. O'Brien, "Dynamics of Charge Collection from 非-Particle Tracks in Integrated Circuits," Proc. IEEE Internationa1 Reliability Physics Symposium, pp. 38-42, Orlando, Florida, Apr 7, 1981.

4. E.L. Petersen, "Nuclear Reactions in Semiconductors," IEEE Trans. Nuc. Sci., NS-27, 1494 (1980).

5. J.A. Adams, Jr., R. Silberberg, and C.H. Tsao, NRL Memo Report 4506 (1981).

6. E.L. Petersen, P. Shapiro, J.H. Adams, Jr., and E.A. Burke, "Calculation of Cosmic-Ray Induced Soft Upsets and Scaling in VLSI Devices," to be published in IEEE Trans. Nuc. Sci., NS-29, Dec 1982.

7. J.C. Pickel and J.T. Blandford, Jr., "CMOS RAM Cosmic-Ray-Induced-Error-Rate Analysis," IEEE Trans. Nuc. Sci., NS-28, 3962 (1981).

8. E.L. Petersen, "Soft Errors Due to Protons in the Radiation Belt," IEEE Trans. Nuc. Sci., NS -28, 3981 (1981).

9. J.C. Pickel and J.T. Blandford, Jr., "Cosmic-Ray-Induced Errors in MOS Devices," IEEE Trans. Nuc. Sci., NS-27, 1006 (1980).

10. J.C. Pickel, "Effect of CMOS Miniaturization on Cosmic-Ray-Induced Error Rate," to be published in IEEE Trans. Nuc. Sci., NS-29, Dec 1982. 